\title{
Correction: Efficacy and Safety of Ipragliflozin in Japanese Patients With Type 2 Diabetes: Interim Outcome of the ASSIGN-K Study
}

Takashi Iizuka ${ }^{a}$, Kotaro Iemitsu ${ }^{a}$, Masahiro Takihata ${ }^{a}$, Masahiko Takai $^{a}$, Shigeru Nakajima ${ }^{a}$,

Nobuaki Minami ${ }^{\text {a }}$, Shinichi Umezawa ${ }^{\text {a }}$, Akira Kanamoria, Hiroshi Takeda ${ }^{\text {a }}$, Takehiro Kawata ${ }^{\text {a }}$ Shogo Ito ${ }^{\text {a }}$ Taisuke Kikuchia ${ }^{a}$ Hikaru Amemiya ${ }^{a}$, Mizuki Kaneshiro ${ }^{a}$, Atsuko Mokubo ${ }^{a}$, Tetsuo Takuma ${ }^{\text {, }}$ Hideo Machimura ${ }^{a}$, Keiji Tanaka ${ }^{a}$, Taro Asakura ${ }^{a}$, Akira Kubota ${ }^{a}$, Sachio Aoyagi ${ }^{a}$, Kazuhiko Hoshino ${ }^{a}$,

Masashi Ishikawa ${ }^{a}$, Yoko Matsuzawa ${ }^{a}$, Mitsuo Obana ${ }^{a}$, Nobuo Sasai ${ }^{a}$, Hideaki Kaneshige ${ }^{a}$, Fuyuki Minagawa $^{a}$, Tatsuya Saito ${ }^{a}$, Kazuaki Shinoda ${ }^{a}$, Masaaki Miyakawa ${ }^{a}$, Yasushi Tanaka ${ }^{a}$,, Yasuo Terauchic, Ikuro Matsuba ${ }^{\mathrm{a}, \mathrm{d}}$

Corrections to article "Efficacy and Safety of Ipragliflozin in Japanese Patients With Type 2 Diabetes: Interim Outcome of the ASSIGN-K Study”, by Takashi Iizuka et al, published in Vol. 8, No. 2, 2016, p116-125, doi: http://dx.doi.org/10.14740/jocmr2417w.

There were some errors in Table 2, the authors would like to make the following corrections.

The baseline postprandial blood glucose level (mg/dL) should read 199.1 \pm 85.2 , instead of $199.1 \pm 1.49$.

The 12 weeks postprandial blood glucose level $(\mathrm{mg} / \mathrm{dL})$ should read $154.9 \pm 60.5$, instead of $54.9 \pm 60.5$.

Manuscript accepted for publication January 20, 2016

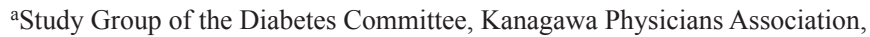
Kanagawa, Japan

${ }^{\mathrm{b} D i v i s i o n}$ of Metabolism and Endocrinology, Department of Internal Medicine, St. Marianna University School of Medicine, Kanagawa, Japan

'Department of Endocrinology and Diabetes, Yokohama City University Medical Center, Kanagawa, Japan

${ }^{\mathrm{d} C}$ Corresponding Author: Ikuro Matsuba, Matsuba Medical Clinic, 2-159 Tsukagoshi, Saiwai-ku, Kawasaki-shi, Kanagawa 212-0024, Japan.

Email: ikuro@matsuba-web.com

doi: http://dx.doi.org/10.14740/jocmr2417wc1

Articles () The authors | Journal compilation (C) Clin Med Res and Elmer Press Inc ${ }^{\top \mathrm{M}} \quad$ | www.jocmr.org

This is an open-access article distributed under the terms of the Creative Commons Attribution License, which permits unrestricted use, distribution, and reproduction

in any medium, provided the original work is properly cited 